\title{
Effect of Chêneau style braces on vertebral wedging amongst individuals with adolescent idiopathic scoliosis
}

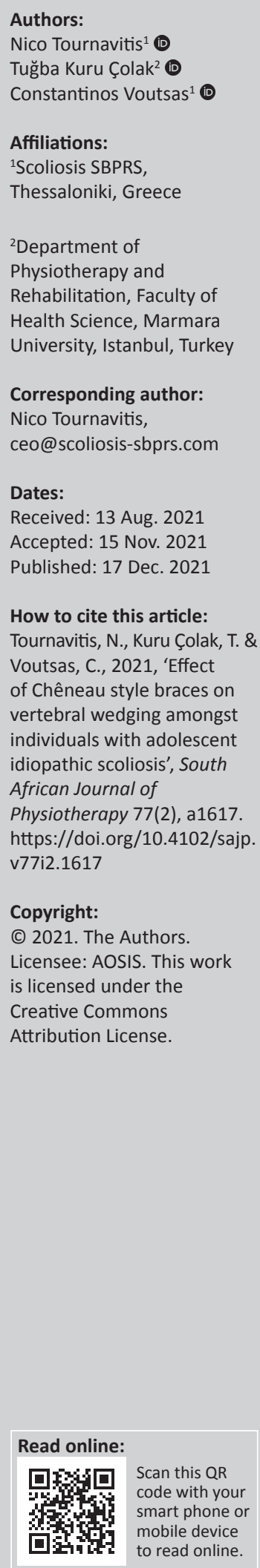

Background: It is generally accepted that braces can stop curve progression but little evidence exists regarding structural improvement in the spine using spinal bracing. Our study aimed to investigate the possible structural improvements of vertebral wedging with high correction bracing.

Objectives: The aim of our study was to assess whether spinal brace treatment may influence vertebral wedging in adolescent idiopathic scoliosis (AIS).

Method: We reviewed our database according to the following inclusion criteria: girls with a diagnosis of AIS, Risser 0-2, age 10-14 years with Cobb angles greater than $35^{\circ}$. Our study cohort consisted of 27 patients fulfilling the inclusion criteria with an average brace wearing time of $16.6 \mathrm{~h}$ per day and Cobb angles between $36^{\circ}$ and $79^{\circ}$. The target value for our study was the apical vertebra wedging, measured twice before brace treatment commenced and twice after the average follow-up period of 20.5 months of treatment.

Results: The average apex wedging noted before brace wearing started was $9.8^{\circ}$ (median: 9) and after a period of 20.5 months of brace wearing, it had reduced to an average of $5.8^{\circ}$ (median: 4.9$),(p<0.001)$. This would indicate a structural correction of $44 \%$.

Conclusions: Our study supports the hypothesis that spinal high correction braces improve the degree of vertebral wedging in skeletally immature girls with AIS.

Clinical implications: Structural corrections of the apical vertebra seem possible when high correction asymmetric braces are used in the treatment of patients with AIS.

Keywords: adolescent; brace; growth; scoliosis; treatment outcome.

\section{Introduction}

Scoliosis is a three-dimensional (3D) deformity of the spine and trunk (Asher \& Burton 2006; Goldberg et al. 2002; Lonstein 1995). Different entities have been described, such as neurogenic, myopathic, congenital types of scoliosis and many others (Chik 2020; Winter 1995). Scoliosis of a known cause may be called symptomatic or syndromic scoliosis (Weiss el al. 2015). Most scoliotic deformities, however, are idiopathic, meaning that there is a deformity in an otherwise healthy child or adolescent (Asher \& Burton 2006; Lonstein 1995). Idiopathic scoliosis tends to progress during phases of rapid growth (Asher \& Burton 2006; Goldberg et al. 2002; Lonstein 1995; Weiss el al. 2015; Winter 1995). Adolescent idiopathic scoliosis (AIS) (which constitutes $80 \%-90 \%$ of all types of scoliosis) is the most common form and appears during adolescence between the ages of 10 and 14 years (Asher \& Burton 2006; Lonstein 1995; Goldberg et al. 2002). Sometimes, progression in AIS curves during the pubertal growth spurt may happen within a matter of a few weeks (Weiss el al. 2015).

The progression in scoliotic curves is usually explained by the vicious cycle concept with an initial asymmetric loading of the vertebra and disc leading to asymmetric growth. This then may trigger further curve progression (Stokes, Burwell \& Dangerfield 2006). However, this concept does not explain why in congenital scoliosis with a calculated risk for progression (Lonstein \& Carlson 1984), the curve may stay stable during growth without any treatment, whilst in a patient with AIS, the curve increases (Weiss \& Moramarco 2016). Obviously, there are other factors leading to progression in AIS. An explanation may be the concept of functional tethering of the spinal cord as outlined in recent literature (Chu et al. 2006; Deng et al. 2015; Weiss, Moramarco \& Borysov 2020).

Nevertheless, when there is a factor triggering curve progression, be it asymmetric loading and/or a functional tether, there may be ways to counteract these forces by correcting the curve 
to reverse this vicious cycle (Landauer, Wimmer \& Behensky 2003; Weiss \& Kleban 2015; Weiss el al. 2015, 2017). In the last decade, this has been undertaken by making use of high correction asymmetric Chêneau style braces (Landauer et al. 2003; Weiss \& Kleban 2015; Weiss el al. 2015, 2017). Although there are authors reporting on an improvement of back shape and trunk (Weiss \& Moramarco 2013; Weiss 2014), as well as authors showing that the angle of curvature can be improved with the help of Chêneau style braces (Landauer et al. 2003; Weiss 2014; Weiss \& Kleban 2015; Weiss \& Moramarco 2013; Weiss el al. 2015, 2017), a systematic investigation of possible structural improvements of vertebral wedging has not yet been undertaken.

Little (2016) has shown that there is an overall pattern of increased disc wedging near the apex of the curvature and reduced joint compliance in this region. Furthermore, Sun (2018) found the same segmental characteristics of main thoracic curves in patients with severe AIS. In the latter study, the apical and adjacent vertebrae accounted for $67.44 \% \pm 8.05 \%$ of the vertebral wedging of the whole curve. During curve progression, if structural changes arise (leading to a wedging of the vertebrae and disc spaces at the apical and adjacent area of the curve), it should be possible to reverse these structural changes in skeletally immature patients with good in-brace correction. The purpose of our study was to investigate if Chêneau style brace treatment may impact vertebral wedging in immature patients with single thoracic curve patterns and curvatures exceeding $35^{\circ}$.

\section{Method}

Our study was conducted in compliance with the Declaration of Helsinki. A parent of each child was informed about the study and written consent was obtained from the participants.

This was a cross-sectional retrospective study, and a quota sampling technique was used to select patients. Patients who were included had participated in Scoliosis Best Practice Rehabilitation Services Thessaloniki, Athens, and Nicosia, between June 2014 and September 2017. Patients were routinely monitored whilst wearing the brace.

Patients enrolled in our study continued to wear a brace after our study was completed. The results of the X-ray examinations of the first and last follow-up are presented here and the $\mathrm{X}$-ray examinations were conducted without the brace being worn.

For each new brace, $\mathrm{X}$-ray check-ups were carried out with the brace being worn for 6 weeks after the adjustment. X-ray examinations were only initiated if a new brace was necessary. All patients included in our study had at least two X-rays without a brace (performed to design new braces). Some patients received three braces maximum.

We reviewed all radiographs of patients with AIS and a single thoracic curve pattern with a Cobb angle (Cobb 1948)
TABLE 1: Demographic and clinical characteristics of patients $(n=27)$

\begin{tabular}{lccc}
\hline Variables & Mean \pm SD & Median & $\begin{array}{c}\text { min-max } \\
(\boldsymbol{n}=\mathbf{2 7} \text { female })\end{array}$ \\
\hline Age (years) & $11.8 \pm 1.1$ & 11.8 & $10.1-13.8$ \\
Risser sign & $0.5 \pm 0.7$ & 0 & $0-2$ \\
$\begin{array}{l}\text { Number of vertebrae in } \\
\text { the curve }\end{array}$ & $6.8 \pm 1.1$ & 7 & $5-9$ \\
Apex of the curve & *T 8.3 \pm 0.8 & T 8 & T 7-10 \\
\hline
\end{tabular}

Source: Table provided by the second author, Tuğba Kuru Çolak.

*T, Thoracic.

greater than $35^{\circ}$ from our clinical database. The demographic characteristics of patients are presented in Table 1. All patients had a standardised computer-aided designed Chêneau style brace (Gensingen brace/GBW ${ }^{\circledR}$, see also Figure 1). Further inclusion criteria were girls having a Risser sign of 0-2 aged 10-14 years with a single thoracic curve pattern. These criteria were selected as single thoracic curve patterns are easier to correct than combined curve patterns (Weiss et al. 2020). Therefore, we estimated that it would be easier to show structural changes in this specific curve pattern. The exclusion criteria were as follows: non-idiopathic scoliosis, neuro-muscular or rheumatic diseases, a previous spinal operation or having received other treatment for scoliosis.

In this curve pattern, there is a long structural C-curve. Long lever arms enable good corrections and may have a beneficial impact on the apical area of the curve. As structural changes are only possible during growth, we only included adolescent patients with the first signs of maturation who were in the pubertal growth spurt.

\section{The brace used}

The brace used for these patients was a Gensingen brace (GBW), which is a Chêneau derivative. Chêneau derivates are asymmetrical trunk orthoses adapted to the corresponding curvature pattern, which today are still often made from a plaster cast. Computer-aided design (CAD)/computer-aided manufacturing (CAM) brace libraries are monitored by experts and continuously being improved, allowing the brace standard to be improved overall (Weiss et al. 2021). Internationally, the GBW use is widespread. The GBW was introduced in 2010 (Weiss 2010) and since 2015 has been used with continuous development and evaluation by international specialists (Weiss et al. 2021) at the first author's centre (Figure 1).

\section{Target value: Apical vertebra wedging measured according to Cobb}

The Cobb method was used to measure the degree of scoliosis: vertical lines were drawn at the upper and lower end vertebra endplate lines on the whole spine anteroposterior (AP) X-ray film and included the angle of the two vertical lines.

The apical vertebra is defined as the most deviated vertebra from the vertical axis that passes through the patient's sacrum on the AP view. The apical vertebra of a scoliotic curve is the most wedged vertebra, and the amount of wedging is bigger in curvatures exceeding $30^{\circ}$ (Modi et al. 2008). The apical 

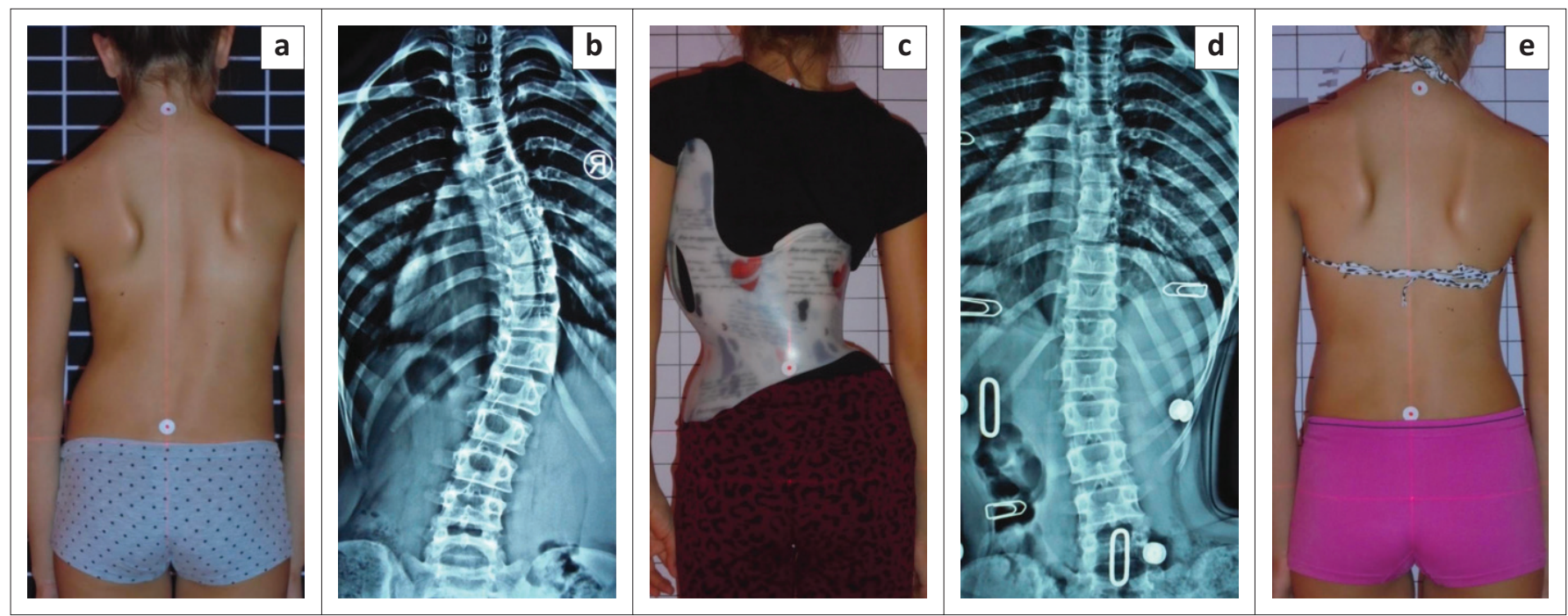

Source: Photos provided courtesy of Nico Tournavitis.

FIGURE 1: Clinical and radiological intermediate result after 6 weeks. From left to right: (a) initial clinical appearance of a single thoracic scoliosis with (b) $44^{\circ}$ of Cobb angle. (c) Patient in brace mirroring of initial deformity is clearly visible. (d) In-brace X-ray with $-17^{\circ}$ of Cobb angle with a (e) clinical over-correction of the pelvic and the trunk.

vertebra wedging (AVW) was determined before starting brace treatment and after the follow-up period.

The AVW angle was measured with the angle formed by two lines connecting the upper and lower endplate of the vertebrae and was made using the Surgimap ${ }^{\circledR}$ software (Modi et al. 2008). All measurements were taken twice by the first author to ensure good intra-rater reliability.

Because the AP measurement of the Cobb angle on an X-ray is a two-dimensional measurement only and because the Cobb angle would be significantly bigger in the 'Plan d'election' (when the scoliotic curve is rotated into the frontal plane; Deacon, Archer \& Dickson 1987; Dickson 2011; Stagnara 1965), a second value might be necessary to exclude any artefacts of measuring by changes in rotation. According to the authors (Deacon et al. 1987; Dickson 2011; Stagnara 1965), the Cobb angle is the biggest when the trunk is turned into the plane of rotation (Plan d'election). This would mean that when rotation is just decreased by simply turning the trunk (Spine) in front of the $\mathrm{X}$-ray detector without changing the Cobb angle, the measurement of the projected Cobb angle would be bigger than in the frontal AP view on the patient's trunk (Dickson 2011). Therefore, a correction of the Cobb angle together with a correction of the rotation can be estimated to be a true reduction of the Cobb angle, not compromised by a rotation artefact.

We only considered readable $X$-rays and measurements taken at the upper and lower endplate of well-defined vertebrae. Therefore, most of the variables contributing to the usual technical error of the Cobb angle measurements do not apply for the measurements taken in our study.

For this reason, we introduced the angle of trunk rotation (ATR) measurements according to Bunnell (2005) with the help of the Scoliometer ${ }^{\mathrm{TM}}$ as a secondary target value (Weiss et al. 2021) to reduce projection artefacts because of an increase or decrease in trunk rotation. The ATR value was obtained in a forward bending position, and the maximum angle as measured was analysed. Given that this was a retrospective study, these measurements were recorded as part of our patients' management programme and then analysed for our study.

\section{Statistical analysis}

Data analysis was performed using Statistical Package for the Social Sciences (SPSS) version 16. The Shapiro-Wilk test was used to test the normality of each variable; $p$-values less than 0.05 were considered to be statistically significant for a two-tailed test. Baseline and last follow-up measurements were compared using the Wilcoxon signed-rank test. Intrarater reliability was established by Spearman's correlation coefficient.

Structural correction was computed simply. The last measured AVW value was subtracted from the first AVW measurement. The percentage of the obtained (result of subtraction) value was calculated.

\section{Results}

A total of 27 female patients from our database fulfilled the inclusion criteria (Figure 2). Patients' mean age was 11.8 years and mean Risser sign value was 0.5 (Table 1 ). Their mean Cobb angle was $43.9^{\circ}$ (range $36^{\circ}-79^{\circ}$ ). The average number of vertebrae in the curve was 6.8 , and the average apex of the curve was $\mathrm{T}$ 8.3. Cobb angle and ATR both improved significantly (Table 2).

Apical vertebra wedging before bracing was $9.8^{\circ}$ and after the monitoring period it was $5.8^{\circ}(p<0.001)$. The monitoring period was an average of 20.5 months (range 11-30 months). These results are statistically significant, demonstrating a structural correction within the apical vertebra of $44 \%$ $(p<0.001)$ (Table 2 and Figure 3 - Figure 5). 
The intra-rater correlation of the measurement of AVW was $r=0.99 ; p<0.05$ with the technical error ranging from $0^{\circ}$ to $1.3^{\circ}$. Therefore, the differences as found at average clearly

\begin{tabular}{|c|c|}
\hline $\begin{array}{c}\text { In } 138 \text { patients with AIS, all } \\
\text { patterns have been analysed } \\
\begin{array}{c}41 \text { patients with a single thoracic } \\
\text { curve pattern: } 3 \text { males and } 38 \\
\text { females }(29.71 \%)\end{array}\end{array}$ & $\begin{array}{l}\text { Excluded: } \\
\text { - Males }(7.32 \%) \text {, (all males had a } \\
\left.\text { Cobb angle greater than } 35^{\circ}\right) \\
\text { - Single thoracic curve pattern with } \\
\text { a Cobb angle smaller than } 35^{\circ} \\
(26.83 \% \text { incl. the three males) }\end{array}$ \\
\hline $\begin{array}{c}\text { Study group: } \\
27 \text { were female and had a single } \\
\text { thoracic curve pattern with a Cobb } \\
\text { angle greater than } 35^{\circ} .71 .05 \% \\
\text { (out of } 38 \text { females) or } 65.85 \% \\
\text { (out of } 41 \text { patients) }\end{array}$ & \\
\hline Analysed: 27 female patients & \\
\hline
\end{tabular}

Source: Figure provided by the second author, Tuğba Kuru Çolak.

AIS, adolescent idiopathic scoliosis.

FIGURE 2: Flow chart of participants. exceed the maximum technical error. The brace wearing time as reported by the patients and their parents was $16.9 \mathrm{~h} /$ day (ranged: 8 to $22 \mathrm{~h}$ ).

\section{Discussion}

Our study results suggest that wearing a reliable high correction Chêneau style brace (Gensingen Brace GBW ${ }^{\circledR}$ ) may lead to the reduction of the structural deformity of the apical vertebrae. This may explain the stable corrections after brace weaning in a high percentage of patients treated with high correction braces (Aulisa et al. 2017; Weiss et al. 2021).

The coincidence of a correction of the Cobb angle and of the rotation (ATR) after the follow-up period again suggests that the improvements of AVW are real structural improvements of the bony shape of the vertebral body and not because of a positional artefact. As the Cobb angle would be significantly bigger in the Plan d'election (when the scoliotic curve is rotated into the frontal plane where the rotation of the apical

TABLE 2: Mean change in Cobb angle, apical vertebra wedging and angle of trunk rotation $(n=27)$.

\begin{tabular}{|c|c|c|c|c|c|c|c|c|c|c|c|}
\hline \multirow[t]{2}{*}{ Variables } & \multicolumn{3}{|c|}{ Before treatment } & \multicolumn{3}{|c|}{ After monitoring period } & \multicolumn{3}{|c|}{ Mean differences } & \multirow[t]{2}{*}{$p$} & \multirow[t]{2}{*}{$Z$} \\
\hline & Mean \pm SD & Median & $\min -\max$ & Mean \pm SD & Median & $\min -\max$ & Mean \pm SD & Median & $\min -\max$ & & \\
\hline Cobb angle $\left({ }^{\circ}\right)$ & $43.9 \pm 12.7$ & 38 & $36-79$ & $34.7 \pm 17.2$ & 34 & $9-74$ & $-9.1 \pm 9.5$ & -7 & $-38-8$ & $<0.001$ & -3.871 \\
\hline $\begin{array}{l}\text { Apical vertebra } \\
\text { wedging }\left({ }^{\circ}\right)\end{array}$ & $9.8 \pm 4.6$ & 9 & $3-19$ & $5.8 \pm 4.2$ & 4.9 & $0.4-14.6$ & $-3.9 \pm 2.6$ & -4.2 & $-10.1-1.0$ & $<0.001$ & -2.480 \\
\hline ATR angle $\left({ }^{\circ}\right)$ & $12.8 \pm 4.9$ & 11 & $7-26$ & $10.6 \pm 5.7$ & 10 & $1-25$ & $-2.1 \pm 4.1$ & -2 & $-13-6$ & 0.013 & -4.382 \\
\hline
\end{tabular}

Source: Table provided by the second author, Tuğba Kuru Çolak.

ATR, angle of trunk rotation.

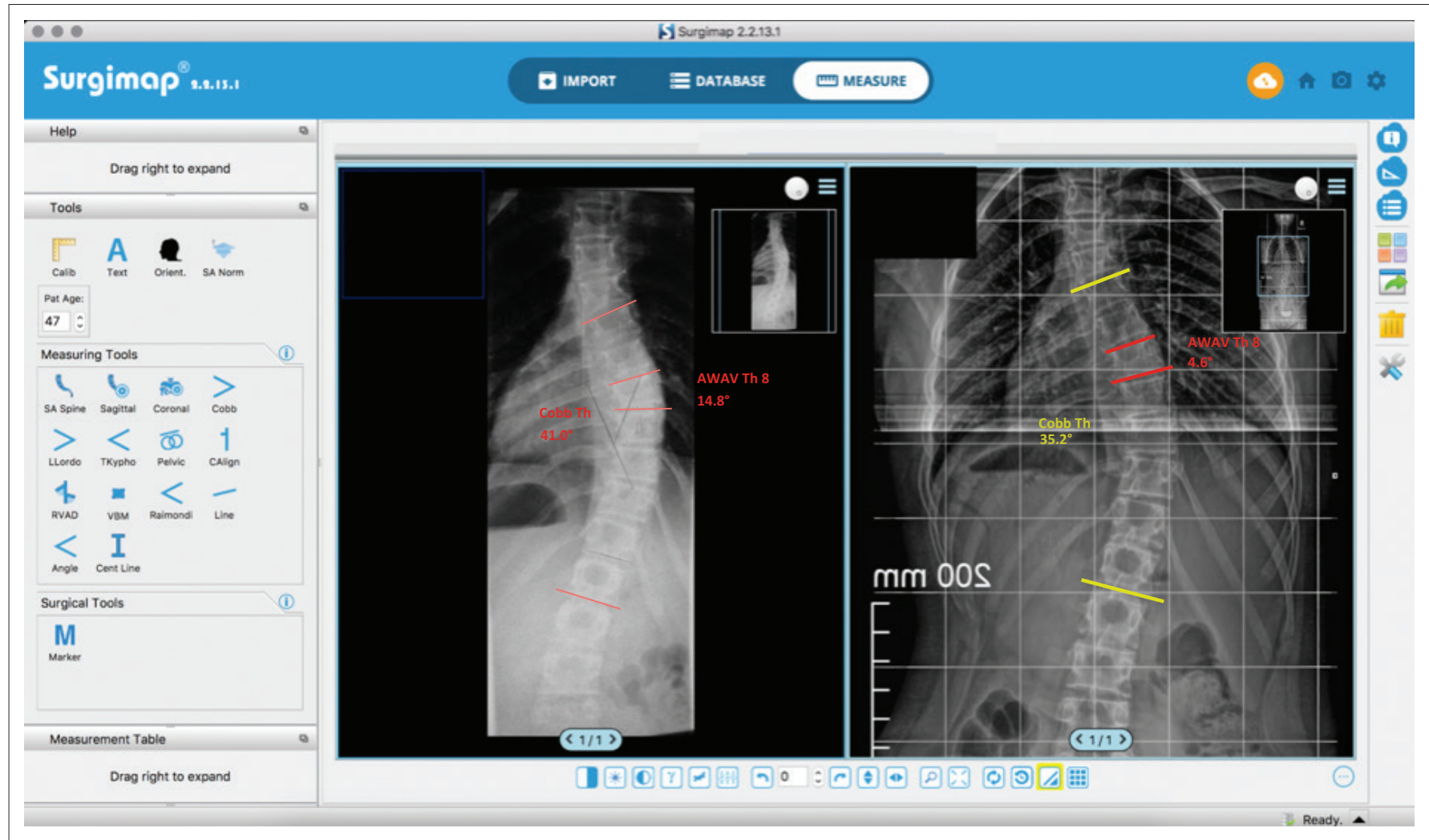

Source: Pictures provided courtesy of the first author, Nico Tournavitis.

FIGURE 3: Reduction of the apical vertebra angle because of the structural correction of the apical and adjacent vertebra after pattern-specific computer-aided design Chêneau style bracing. A reduction has been found in this case. The apical vertebra decreased from $14.8^{\circ}$ to $4.6^{\circ}$ after 11 months of treatment (Risser 0 ). The most wedged vertebrae were above the apex in this case. 


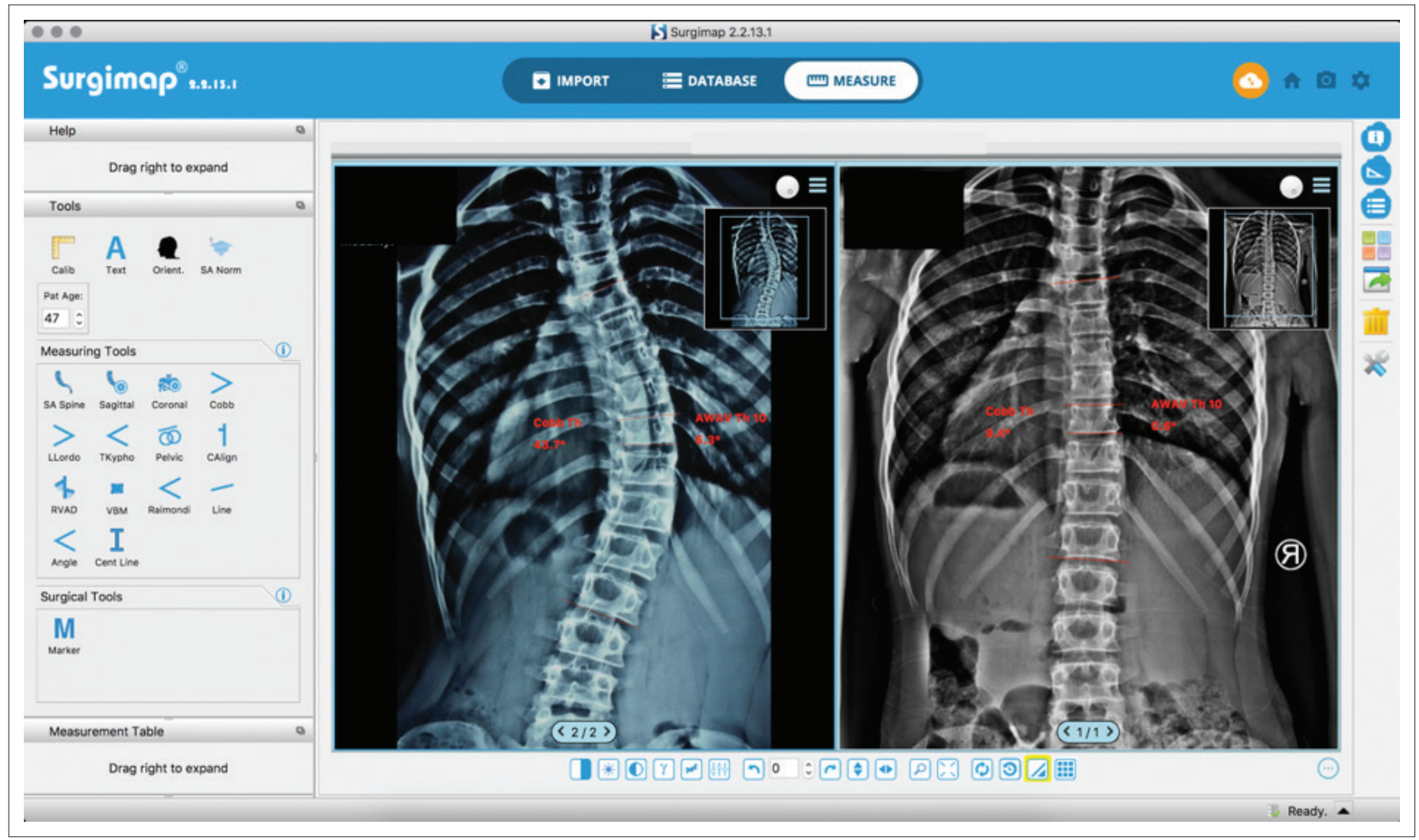

Source: Pictures provided courtesy of the first author, Nico Tournavitis.

FIGURE 4: This case shows that with highly corrective asymmetric Chêneau style braces (Gensingen Brace ${ }^{\circledR}$ ) structural corrections are possible and favourable final outcomes for the patient can be achieved. Almost complete restoration with the wedged vertebra changing from $6.3^{\circ}$ to $0.6^{\circ}$ after 10.5 months of treatment (Risser 0).

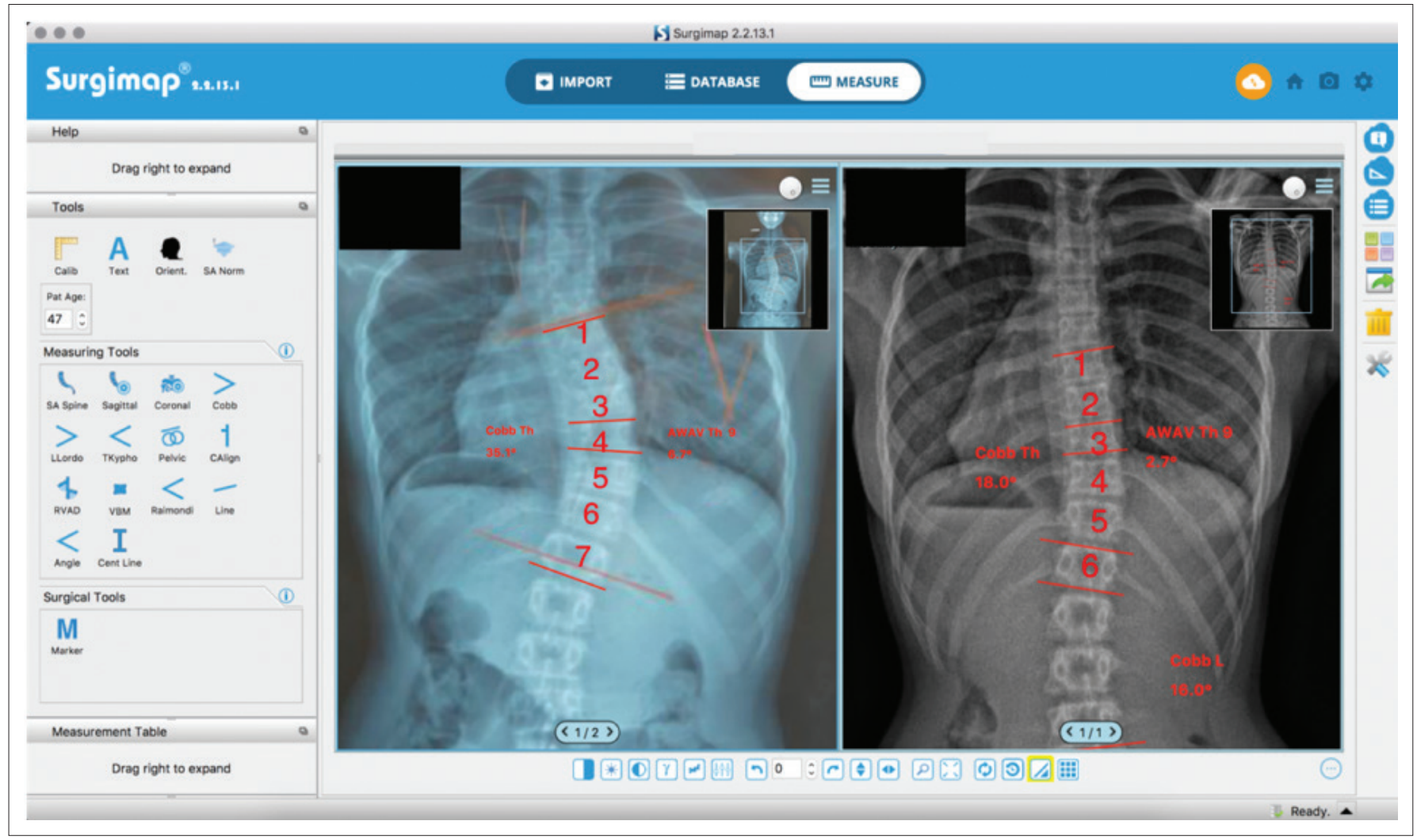

Source: Pictures provided courtesy of the first author, Nico Tournavitis.

FIGURE 5: Reduced number of vertebrae affected after 14 months of treatment, from seven to six vertebrae (Risser 0 ) within the curve. 
vertebra would be $0^{\circ}$; Deacon et al. 1987; Dickson 2011; Stagnara 1965), the Cobb angle measurement would be expected to be smaller when the scoliotic curve is measured in the standard AP X-ray position whilst the rotation of the apical vertebra increases. Therefore, when there is simultaneously a decrease in rotation and a decrease in Cobb angle, we may conclude that a real structural improvement has been achieved, not compromised by a rotational artefact.

The vertebral wedging is seen as the most important factor in progression of scoliosis by some researchers (Braun et al. 2003; Perdriolle et al. 1993). The latter authors (Perdriolle et al. 1993) have underlined that vertebral wedging is not only the most essential deformation process, but also has a direct correlation with the curve magnitude. According to the Hueter-Volkmann law (Mehlman, Araghi \& Roy 1997), compressive forces inhibit the epiphyseal growth, and distraction forces stimulate the epiphyseal growth. Based on this law, Roaf (1960) has suggested a 'vicious cycle' concerning kyphosis progression. According to this concept, a minimal wedging of the vertebrae would produce asymmetric compressive force on the vertebral end plates, which would further increase the wedging as per the Hueter-Volkmann's law and thus produce further abnormal forces. For this reason, it is important to control vertebral wedging within the conservative management of spinal deformities.

We analysed the radiographs of patients with Cobb angles exceeding $35^{\circ}$, and the mean AWV value was $9.8^{\circ}$ before treatment. Modi et al. (2008) evaluated the radiographs of patients with AIS with a Cobb angle of $10^{\circ}-60^{\circ}$ (mean: 27.1) and an age range of $11-20$ years, and reported $4.1^{\circ}$ of vertebral body wedging in the thoracic spines of patients with Cobb angles above $30^{\circ}$. However, the authors did not provide a study in a pre-/post-design outlining an effect of any treatment. The difference in AWV between our study and that of Modi et al. (2008) could be explained by the difference in the mean Cobb angles. To the best of our knowledge, there is no study investigating the effect of brace treatment on vertebral wedging.

Structural corrections of the apical vertebra seem possible when high correction asymmetric braces are used in the treatment of patients with AIS. Structural corrections of the apical and adjacent vertebrae have a favourable influence on the outcome for the patient. It has been found that in a reasonable percentage of patients treated with a high correction brace, final improvements can be achieved (Landauer et al. 2003; Weiss 2014; Weiss \& Kleban 2015; Weiss \& Moramarco 2013; Weiss, Seibel \& Kleban 2014; Weiss et al. 2017, 2019; ). As AIS does not lead to severe health problems besides the deviation (Weinstein, Zavala \& Ponseti 1981; Weinstein et al. 2003; Weiss et al. 2016), the focus in future studies should shift from aiming at improvements of Cobb angle to improvement of the cosmetic outcomes and how different braces may influence these in the long term.

We used a standardised computer-aided designed Chêneau style brace $\left(\mathrm{GBW}^{\circledR}\right)$. In a certain percentage of patients using the GBW, improvements of their spinal deformity have been noted in addition to improvement of the Cobb angle (Weiss \& Moramarco 2013; Weiss 2014; Weiss et al. 2014, 2019). Within this investigation, we found structural changes explaining the improvements obtained in Cobb and ATR angles.

Our study focused on one single curve pattern selectively to see whether structural changes are possible at all when applying asymmetric braces. In a study by Borysov et al. (2013), the in-brace correction in double curve patterns was less than that in single curve patterns. Therefore, in future studies, the effect on bracing in combined curves should be investigated.

For symmetric braces (Weinstein et al. 2013), structural remodelling effects have not been found. Therefore, we would suggest applying standardised CAD Chêneau derivates wherever possible (Rigo 1999, 2003; Rigo \& Weiss 2003; Weiss 2014; Weiss \& Moramarco 2013; Weiss et al. 2017).

The technical error (variability of the measurements) of the Cobb angle measurement lies between $3^{\circ}$ (Wang et al. 2018) and $6^{\circ}$ (Loder et al. 2004). Therefore, measurement differences of $\pm 5^{\circ}$ are defined as unchanged, whilst for the definition of improvement or progression, a difference of $6^{\circ}$ or more Cobb angle is defined. This would be appropriate for measuring whole curvatures involving six or more vertebrae. Most of the technical error may be because of the variability of segment selection, variability in the selection of upper or lower endplate of the vertebra and lack of readability of the X-ray (quality of the picture).

Studies evaluating the effect of brace treatment on AVW in patients with AIS are very limited. Presenting the results of AVW assessment in patients with a specific curve pattern and continued growth and with risk of progression is the strength of our study.

The lack of blind assessors collecting outcome data and comparison of the effect of different brace types on AVW is a limitation of our study. Secondly, the AVW as measured according to Cobb on an AP X-ray is a measurement in one plane only and does not describe the true $3 \mathrm{D}$ character of a scoliotic deformity. A complete 3D analysis would only be possible with the help of the $3 \mathrm{D}$ reconstruction of a computer tomography scan. However, the radiation exposure resulting from this examination does not seem appropriate to the question of our examination.

\section{Conclusion}

Our study supports the hypothesis that spinal high correction braces may improve the degree of vertebral wedging in skeletally immature girls with AIS.

\section{Acknowledgements}

The authors would like to thank all patients and their parents for having agreed to publication of their pictures and X-rays within scientific articles. 


\section{Competing interests}

The first author currently serves as a certified orthotist. The co-authors declare that they have no financial or personal relationships that may have inappropriately influenced them in writing this article.

\section{Authors' contributions}

N.T. was responsible for the conception, literature review and first draft of the article. T.K.C. contributed towards the conception, data analysis, editing and review of the article. C.V. was responsible for the conception and data analysis of the study.

\section{Ethical considerations}

This article followed all ethical standards for research without direct contact with human or animal subjects.

\section{Funding information}

This research received no specific grant from any funding agency in the public, commercial or not-for-profit sectors.

\section{Data availability}

Data sharing is not applicable to this article as no new data were created or analysed in this study.

\section{Disclaimer}

The views and opinions expressed in this article are those of the authors and do not necessarily reflect the official policy or position of any affiliate agency of the authors.

\section{References}

Asher, M.A. \& Burton, D.C., 2006, 'Adolescent idiopathic scoliosis: Natural history and long term treatment effects', Scoliosis 1(1), 2. https://doi.org/10.1186/1748-7161-1-2

Aulisa, A.G., Guzzanti, V., Falciglia, F., Galli, M., Pizzetti, P. \& Aulisa, L., 2017, ‘Curve progression after long-term brace treatment in adolescent idiopathic scoliosis: Comparative results between over and under 30 Cobb degrees - SOSORT 201 award winner', Scoliosis and Spinal Disorders 12, 36. https://doi.org/10.1186/ s13013-017-0142-y

Borysov, M., Borysov, A., Kleban, A. \& Weiss, H.R., 2013, 'Bracing according to best practice standards - Are the results repeatable?', Orthopedic \& Muscular System: Current Research 01(S1), 006. https://doi.org/10.4172/2161-0533.s1-006

Braun, J.T., Ogilvie, J.W., Akyuz, E., Brodke, D.S., Bachus, K.N. \& Stefko, R, 2003, 'Experimental scoliosis in an immature goat model: A method that creates idiopathictype deformity with minimal violation of the spinal elements along the curve', Spine 28(19), 2198-2203. https://doi.org/10.1097/01.BRS.0000085095.37311.46

Bunnell, W.P., 2005, 'Selective screening for scoliosis', Clinical Orthopaedics and Related Research 434, 40-45. https://doi.org/10.1097/01.blo.0000163242.92733.66

Chik, S.K.T., 2020, 'Classification and terminology', in M. Moramarco, M. Borysov, S.Y, Ng \& H.R. Weiss (eds.), Schroth's textbook of scoliosis and other spinal deformities, pp 150-158, Cambridge Scholars Publishing, Newcastle upon Tyne.

Chu, W.C.W., Lam, W.W.M., Chan, Y.-L., Ng, B.K.W., Lam, T.-P., Lee, K.-M. et al., 2006 'Relative shortening and functional tethering of spinal cord in adolescent idiopathic scoliosis? Study with multiplanar reformat magnetic resonance imaging and somatosensory evoked potential', Spine 31(1), E19-E25. https://doi. arg $\mathrm{a}$ o.1097/01.brs.0000193892.20764.51

Cobb, J.R., 1948, 'Outline for the study of scoliosis', Instructional Course Lectures 5, 261-275

Deacon, P., Archer, I.A. \& Dickson, R.A., 1987, 'The anatomy of spinal deformity: A biomechanical analysis', Orthopedics 10, 897-903. https://doi.org/10.3928/ 0147-7447-19870601-09

Deng, M., Hui, S.C.N., Fu, Y.W.P., Ng, B.K.W., Lam, T.-P., Qiu, Y. et al., 2015, 'MRI-based morphological evidence of spinal cord tethering predicts curve progression in adolescent idiopathic scoliosis', The Spine Journal: Official Journal of the North American Spine Society 15(6), 1391-1401. https://doi.org/10.1016/j.spinee.2015. 02.033
Dickson, R.A., 2011, 'Pathogenesis of idiopathic scoliosis', in P.O. Newton, M.F. O'Brien, H.L. Shufflebarger, et al. (eds.), Idiopathic scoliosis - The Harms Study Group Treatment Guide, pp. 28-50, Thieme, Stuttgart. Print ISBN: 9781604060249 ; Group Treatment Guide, pp. 28

Goldberg, C.J., Moore, D.P., Fogarty, E.E. \& Dowling, F.E., 2002, 'Adolescent idiopathic scoliosis: natural history and prognosis', Studies in health technology and informatics 91, 59-63.

Landauer, F., Wimmer, C. \& Behensky, H., 2003, 'Estimating the final outcome of brace treatment for idiopathic thoracic scoliosis at 6-month follow-up', Pediatric Rehabilitation 6(3-4), 201-207. https://doi.org/10.1080/1363849031 0001636817

Little, J.P., 2016, 'Understanding how axial loads on the spine influence segmental biomechanics for idiopathic scoliosis patients: A magnetic resonance imaging study', Clinical biomechanics (Bristol, Aon), 32, 220-228. https://doi. study', Clinical biomechanics (Bristol,
org/10.1016/j.clinbiomech.2015.11.007

Loder, R.T., Spiegel, D., Gutknecht, S., Kleist, K., Ly, T. \& Mehbod, A., 2004, 'The assessment of intra observer and inter observer error in the measurement of noncongenital scoliosis in children <or=10 years of age', Spine 29(22). https://doi. org/10.1097/01.brs.0000144828.72721.d8

Lonstein, J. 1995, 'Idiopathic scoliosis', in J.E. Lonstein, D.S. Bradford, R.B. Winter \& J.W. Ogilvie (eds.), Moe's Textbook of Scoliosis and other Spinal Deformities, 3rd edn., pp. 219-256, WB Saunders, Philadelphia, PA.

Lonstein, J.E. \& Carlson, J.M., 1984, 'The prediction of curve progression in untreated idiopathic scoliosis during growth', The Journal of Bone and Joint Surgery. American volume 66(7), 1061-1071. https://doi.org/10.2106/00004623-19846607000013

Mehlman, C.T., Araghi, A. \& Roy, D.R., 1997, 'Hyphenated history: The HueterVolkmann law', American Journal of Orthopedics (Belle Mead, N.J.) 26(11) 798-800.

Modi, H.N., Suh, S.W., Song, H.-R., Yang, J.-H., Kim, H.J., Modi, V.H. et al., 2008, 'Differential wedging of vertebral body and intervertebral disc in thoracic and lumbar spine in adolescent idiopathic scoliosis - A cross sectional study in 150 patients', Scoliosis 3(1), 11. https://doi.org/10.1186/1748-7161-3-11

Perdriolle, R., Becchetti, S., Vidal, J. \& Lopez, P., 1993, 'Mechanical process and growth cartilages. Essential factors in the progression of scoliosis', Spine 18(3), 343-349. https://doi.org/10.1097/00007632-199303000-00007

Rigo, M., 1999, '3 D correction of trunk deformity in patients with idiopathic scoliosis using Chêneau Brace', in I.A.F. Stokes (ed.), Research into Spinal Deformities 2 pp. 362-365, IOS Press, Amsterdam.

Rigo, M., 2003, 'Radiological and cosmetic improvement 2 years after brace weaning A case report', Pediatric Rehabilitation 6(3-4), 195-199. https://doi.org/10.1080/ 13638490310001636826

Rigo, M. \& Weiss, H.R., 2003, 'Korsettversorgungsstrategien in der Skoliosebehandlung', in H.R. Weiss (ed.), Wirbelsäulendeformitäten - Konservatives Management, pp. 251-286, Pflaum, Munich.

Roaf, R., 1960, 'Vertebral growth and its mechanical control', The Journal of Bone and Joint Surgery. British Volume 42-B(1), 40-59. https://doi.org/10.1302/0301-620X. 42B1.40

Stagnara, P., 1965, 'Le plan d'election pour l'examen radiologique des cyphoscolioses', Rev Chir Orthop Reparatrice Appar Mot 51, 517-524.

Stokes, I.A.F., Burwell, R.G. \& Dangerfield, P.H. 2006, 'Biomechanical spinal growth modulation and progressive adolescent scoliosis - A test of the "vicious cycle" pathogenetic hypothesis: Summary of an electronic focus group debate of the IBSE', Scoliosis 1(1), 16. https://doi.org/10.1186/1748-7161-1-16

Sun, X., 2018, 'Segmental characteristics of main thoracic curves in patients with severe adolescent idiopathic scoliosis', World Neurosurgery 119, 174-179. https://doi.org/10.1016/j.wneu.2018.07.086

Wang, J., Zhang, J., Xu, R., Ge Chen, T., Zhou, K.S. \& Zhang, H.H., 2018, 'Measurement of scoliosis Cobb angle by end vertebra tilt angle method', Journal of Orthopaedic Surgery and Research 13(1), 223. https://doi.org/10.1186/s13018-018-0928-5

Weinstein, S.L., Dolan, L.A., Spratt, K.F., Peterson, K.K., Spoonamore, M.J. \& Ponseti, I.V., 2003, 'Health and function of patients with untreated idiopathic scoliosis: A 50-year natural history study', JAMA 289(5), 559-567. https://doi.org/10.1001/ jama.289.5.559

Weinstein, S.L., Dolan, L.A., Wright, J.G. \& Dobbs, M.B., 2013, 'Effects of bracing in adolescents with idiopathic scoliosis', The New England Journal of Medicine 369(16), 1512-1521. https://doi.org/10.1056/NEJMoa1307337

Weinstein, S.L., Zavala, D.C. \& Ponseti, I.V., 1981, 'Idiopathic scoliosis: Long-term follow-up and prognosis in untreated patients', The Journal of Bone and Joint Surgery. American Volume 63(5), 702-712. https://doi.org/10.2106/00004623198163050-00003

Weiss, H.R., 2010, “"Brace technology” thematic series - The Gensingen brace ${ }^{\mathrm{TM}}$ in the treatment of scoliosis', Scoliosis 5, 22. https://doi.org/10.1186/1748-7161-5-22

Weiss, H.R., 2014, 'Bracing can lead to a persistent correction in the treatment of Adolescent Idiopathic Scoliosis: A case report', Hard Tissue 3(1), 8.

Weiss, H.R., Karavidas, N., Moramarco, M. \& Moramarco, K. 2016, 'Long-term effects of untreated adolescent idiopathic scoliosis: A review of the literature', Asian Spine Journal 10(6), 1163-1169. https://doi.org/10.4184/asj.2016.10. 6.1163

Weiss, H.R., \& Kleban, A., 2015, 'Development of CAD/CAM Based Brace Models for the Treatment of Patients with Scoliosis-Classification Based Approach versus Finite Element Modelling', Asian spine journal 9(5), 661-667. https://doi. org/10.4184/asj.2015.9.5.661 
Weiss, H.R., Lay, M., Seibel, S. \& Kleban A., 2021, 'Ist eine Verbesserung der Behandlungssicherheit in der Korsettversorgung von Skoliosepatienten durch Behandlungsicherheit in der Korsettversorgung von Skoliosepatienten durch
Anwendung standardisierter CAD-Algorithmen möglich? [Is it possible to improve Anwendung standardisierter CAD-Algorithmen moglich? [Is it possible to improve
treatment safety in the brace treatment of scoliosis patients by using standardized treatment safety in the brace treatment of scoliosis patients by using standardized
CAD algorithms?]', Der Orthopade 50(6), 435-445. https://doi.org/10.1007/ CAD algorithms?]', Der

Weiss, H.R. \& Moramarco, M., 2013, 'Remodelling of trunk and backshape deformities in patients with scoliosis using standardized asymmetric computer-aided design/ computer-aided manufacturing braces', Hard Tissue 2(2), 14. https://doi. computer-aided manufacturing
org/10.13172/2050-2303-2-2-397

Weiss, H.R. \& Moramarco, M., 2016, 'Congenital scoliosis (mini review)', Current Pediatric Reviews 12(1), 43-47. https://doi.org/10.2174/1573396312666151117121011

Weiss, H.R., Moramarco, M. \& Borysov, M., 2020, 'Physical rehabilitation and exercise', in M. Moramarco, M. Borysov, S.Y. Ng \& H.R. Weiss(eds.), Schroth's textbook of scoliosis and other spinal deformities, pp. 632-658, Cambridge Scholars Publishing, Newcastle upon Tyne.
Weiss, H.R., Seibel, S. \& Kleban, A., 2014, 'Deformity-related stress in a sample of patients with adolescent idiopathic scoliosis (AIS) after brace weaning: A crosssectional investigation', OA Musculoskeletal Medicine 2(1), 5.

Weiss, H.R., Tournavitis, N., Seibel, S. \& Kleban, A., 2017, 'A prospective cohort study of AIS patients with $40^{\circ}$ and more treated with a Gensingen brace (GBW): Preliminary results', The Open Orthopaedics Journal 11, 1558-1567. https://doi. org/10.2174/1874325001711011558

Weiss, H.-R., Turnbull, D., Seibel, S. \& Kleban, A., 2019, 'First end-result of a prospective cohort with AIS treated with a CAD Chêneau style brace', Journal of Physical Therapy Science 31(12), 983-991. https://doi.org/10.1589/jpts.31.983

Weiss, H.-R., Lehnert-Schroth, C., Moramarco, M. \& Moramarco, K., 2015, Advancements in conservative scoliosis treatment lambert, Academic Publishing, Saarbruecken.

Winter, R., 1995, 'Classification and terminology', in J.E. Lonstein, D.S. Bradford, R.B. Winter \& J.W. Ogilvie (eds.), Moe's Textbook of Scoliosis and other Spinal Deformities, 3rd edn., pp. 39-44, WB Saunders, Philadelphia. 